\title{
Oxigenoterapia de alto fluxo nasal em paciente com fibrose pulmonar idiopática sob cuidados paliativos: relato de um caso
}

\section{Oxygen high flow nasal in patient with pulmonary fibrosis idiopathic in palliative care: report of a case}

Francisca Soraya Lima Silva1. Patriciane Hedwiges Barreto ${ }^{1}$. Renata dos Santos de Vasconcelos ${ }^{2}$. Raquel Pinto Sales ${ }^{3}$. Andréa da Nóbrega Cirino Nogueira ${ }^{4}$. Marcelo Alcantara Holanda ${ }^{5}$.

1 Fisioterapeuta, Residência em Terapia Intensiva no Hospital Universitário Walter Cantídio (HUWC), Universidade Federal do Ceará (UFC), Fortaleza, Ceará, Brasil. 2 Fisioterapeuta, Doutoranda em ciências médicas pela Universidade Federal do Ceará (UFC), coordenadora das UTI's do Hospital Universitário Walter Cantídio (HUWC), Fortaleza, Ceará, Brasil. 3 Mestre em ciências médicas pela Universidade Federal do Ceará (UFC), Fisioterapeuta da Empresa Brasileira de Serviços Hospitalares (EBSERH), Fortaleza, Ceará, Brasil. 4 Fisioterapeuta, Doutoranda em Ciências médicas pela Universidade Federal do Ceará (UFC), coordenadora da Residência Integrada Multiprofissional em Atenção Hospitalar da UFC, Fortaleza, Ceará, Brasil. 5 Médico, Doutor em Pneumologia pela Universidade Federal de São Paulo (UNIFESP), professor associado de Terapia Intensiva e Pneumologia do Departamento de Medicina Clínica da Universidade Federal do Ceará (UFC), Fortaleza, Ceará, Brasil.

\section{RESUMO}

Objetivo: avaliar o uso da terapia de Alto Fluxo Nasal (AFN) em uma paciente com Fibrose Pulmonar Idiopática (FPI) sob cuidados paliativos. Relato de caso: mulher de 70 anos, admitida em uma Unidade de Terapia Intensiva (UTI) por insuficiência respiratória hipoxêmica grave por FPI em estágio avançado, sem indicação de intubação traqueal. Inicialmente tratada com Ventilação Não Invasiva (VNI) intercalando com máscara reservatório de oxigênio $\left(\mathrm{O}_{2}\right)$, porém com baixa tolerância. Mesmo com a máscara reservatório de $\mathrm{O}_{2}$ apresentou frequência respiratória (f) de 40rpm, saturação periférica de $\mathrm{O}_{2}\left(\mathrm{SpO}_{2}\right)$ de $58 \%$, frequência cardíaca (FC) de 135 bpm, pressão arterial (PA) $175 \times 85 \mathrm{mmHg}$. A gasometria arterial revelou $\mathrm{PaO}_{2}$ de $36 \mathrm{mmHg}$ e $\mathrm{SaO}_{2}$ de $72 \%$. Optou-se por usar o AFN pelo sistema Optiflow ${ }^{\mathrm{TM}}$ Fisher \& Paykel $^{\circledR}$ com fluxo de $30 \mathrm{l} / \mathrm{min}$ de $\mathrm{O}_{2}$ e temperatura de $37^{\circ} \mathrm{C}$. Após 30 minutos houve resposta clínica imediata, apresentou: $\mathrm{f}=30 \mathrm{rpm}, \mathrm{FC}=127 \mathrm{bpm}, \mathrm{PA}=156 \times 69 \mathrm{mmHg}, \mathrm{SpO}_{2}=80 \%, \mathrm{PaO}_{2}=46 \mathrm{mmHg} \mathrm{SaO}_{2}$ $=83 \%$. Após 24 horas apresentou $\mathrm{PaO}_{2}=56 \mathrm{mmHg}$ e $\mathrm{SaO}_{2}=90 \%$, sem alteração de $\mathrm{pH}$. Conclusão: o AFN foi importante para o melhora da oxigenação e conforto da paciente do caso relatado, garantindo além da melhora clínica, uma morte tranquila com a redução dos sintomas respiratórios.

Palavras-chave: Oxigenoterapia. Fibrose pulmonar. Cuidados paliativos.

\section{ABSTRACT}

Objective: to evaluate the use of the High-therapy Nasal Flow (AFN) in a patient with Idiopathic Pulmonary Fibrosis (IPF) in palliative care. Case report: a 70-year-old admitted to the ICU for severe hypoxic respiratory failure by FPI in an advanced stage, with no indication of tracheal intubation. Initially treated with Non-Invasive Ventilation (NIV) interspersed with mask oxygen tank $\left(\mathrm{O}_{2}\right)$, but with low tolerance. Even with the mask $\mathrm{O}_{2}$ reservoir presented respiratory frequency (f) of 40rpm, peripheral $\mathrm{O}_{2}$ saturation $\left(\mathrm{SpO}_{2}\right)$ of $58 \%$, heart rate (HR) of $135 \mathrm{bpm}$, blood pressure (BP) $175 \times 85 \mathrm{mmHg}$. Arterial blood gas analysis showed $\mathrm{PaO}_{2} 36 \mathrm{mmHg}$ and $\mathrm{SaO}_{2}$ of $72 \%$. We chose to use the AFN by Optiflow ${ }^{\mathrm{TM}}$ Fisher \& $\mathrm{Payke}^{\circledR}$ system with $30 \mathrm{l} / \mathrm{min}_{2}$ and $37^{\circ} \mathrm{C}$. After 30 minutes there was immediate clinical response, showed: $\mathrm{f}=30 \mathrm{rpm}, \mathrm{HR}=127 \mathrm{bpm}, \mathrm{BP}=156 \times 69 \mathrm{mmHg}, \mathrm{SpO}_{2}=80 \%$, $\mathrm{PaO}_{2}=46 \mathrm{mmHg}$ and $\mathrm{SaO}_{2}=83 \%$. After 24 hours showed $\mathrm{PaO}_{2}=56 \mathrm{mmHg}$ and $\mathrm{SaO}_{2}=90 \%$, no change of pH. Conclusion: the AFN was important for the improved oxygenation and comfort of the case reported, guaranteeing beyond clinical improvement, a peaceful death with the reduction in respiratory symptoms.

Keywords: Oxigen inhalation therapy. Pulmonary fibrosis. Palliative care.

Autor correspondente: Francisca Soraya Lima Silva, Rua 15, 69 A, Arvoredo, Mondubim, Fortaleza, Ceará. CEP: 60.753-450.Telefone: +55 85 98605-4612. E-mail: soraya.lima2@gmail.com

Conflito de interesses: Não há qualquer conflito de interesses por parte de qualquer um dos autores.

Recebido em: 28 Jun 2015; Revisado em: 25 Ago 2016; Aceito em: 30 Ago 2016. 


\section{INTRODUÇÃO}

A fibrose pulmonar idiopática (FPI) é a mais comum das pneumopatias intersticiais idiopáticas e carrega o pior prognóstico. Sua prevalência, estimada em torno de 30 casos por 100.000 pessoas, aumenta vertiginosamente com a idade, afetando mais de 100 indivíduos por 100.000 pessoas com idade igual ou superior a 75 anos. Os sintomas incluem tosse seca e falta de ar progressiva. Uma sobrevida de aproximadamente três anos, a partir do diagnóstico, pode ser esperada. ${ }^{1}$

A incidência de insuficiência respiratória em fibrose pulmonar é alta, e esta é a causa mais comum de morte nesses pacientes. O mau prognóstico e a redução da qualidade de vida são resultados de necessidade de cuidados paliativos. ${ }^{2}$

Os pacientes portadores de doenças sem possibilidades de continuidade da vida ou de cura, como as doenças graves, progressivas e incuráveis, devem ser abordados com cuidados paliativos. Dentre os princípios dessa abordagem estão o alívio da dor e de outros sintomas desagradáveis, não acelerar nem adiar a morte, cuidado multiprofissional aos pacientes e seus familiares, controlar situações clínicas estressantes e melhorar a qualidade de vida. ${ }^{3}$

Dentre os sintomas presentes nos indivíduos em cuidados paliativos a dispneia tem maior incidência, sendo esta descrita como uma sensação subjetiva de desconforto respiratório tratando-se de um sintoma desagradável e estressante que interfere diretamente na qualidade de vida. ${ }^{4}$

O oxigênio está entre abordagens utilizadas para a redução da dispneia nos cuidados paliativos. Indicado nos indivíduos com hipoxemia grave, a oxigenoterapia favorece a redução da intensidade da dispneia, redução do trabalho respiratório, otimização da função cardiovascular, melhora do desconforto respiratório e do quadro clínico. ${ }^{5}$

O uso contínuo de oxigênio pode resultar em ressecamento da região nasal, oral e redução da interação do indivíduo com seus familiares e equipe prejudicando a socialização. Diante disso a oxigenoterapia com uso de cânulas nasais com fluxos umidificados e aquecidos vem demonstrando resultados positivos na redução da frequência respiratória e aumento da saturação, trazendo benefícios aos pacientes que apresentam dispneia em repouso e hipoxemia. ${ }^{6}$

$\mathrm{Na}$ oxigenoterapia de alto fluxo por cânula nasal o gás fornecido umidificado e aquecido proporciona maior conforto e aceitação de altos fluxos de oxigênio, podendo este ser ofertado em até 60 1/min com Fração Inspirada de Oxigênio $\left(\mathrm{FiO}_{2}\right)$ de até $100 \%$. Essa terapia vem apresentando resultados satisfatórios na melhora das dificuldades respiratórias, reduzindo a necessidade de intubação endotraqueal e internação nas unidades de terapias intensivas (UTI's), é bem tolerada e segura em pacientes conduzidos em cuidados paliativos, pois proporciona redução do desconforto respiratório. ${ }^{7}$

Diante do exposto avaliamos o uso da terapia de Alto
Fluxo Nasal (AFN) em uma paciente com fibrose pulmonar idiopática sob cuidados paliativos.

\section{RELATO DE CASO}

Este estudo foi aprovado pelo comitê de ética em pesquisa segundo parecer número 1.359.112.

Trata-se de uma paciente de 70 anos, sexo feminino, procedente da cidade de Fortaleza-CE, admitida em uma unidade hospitalar privada, apresentando desconforto respiratório por Insuficiência Respiratória Aguda (IRpA) hipoxêmica devido à FPI em estágio avançado (Figura 1). Como queixa principal relatava dispneia e constipação. Devido ao estágio avançado da fibrose pulmonar a paciente passou a receber cuidados paliativos com orientação para não intubá-la e não reanimá-la.

Figura 1. Imagem radiológica do tórax na admissão. Redução acentuada dos volumes pulmonares com opacidade reticulo-nodulares bilaterais, alargamento e distorção da traqueia.

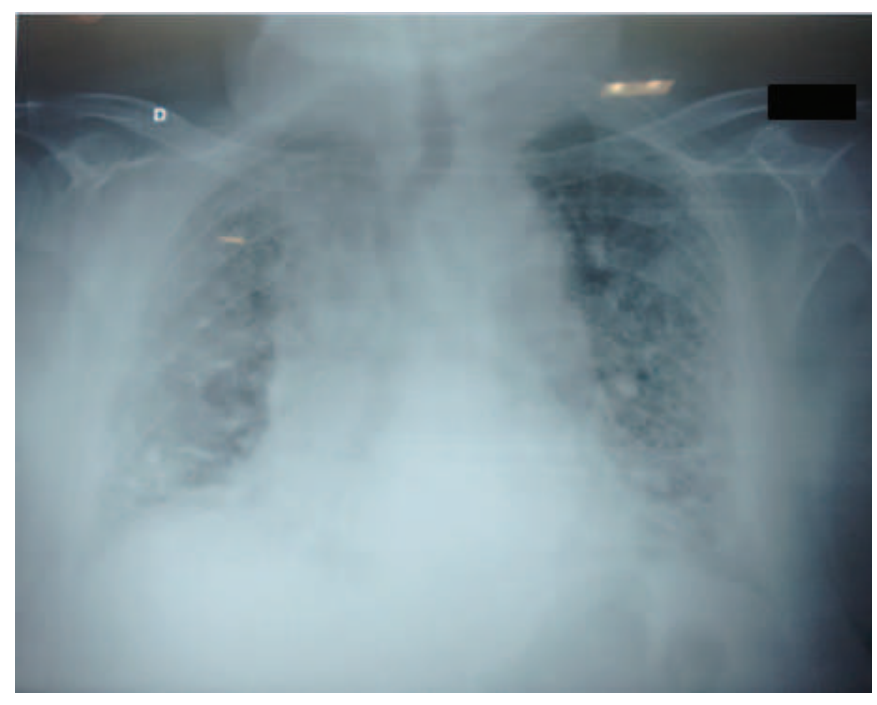

A conduta adotada para diminuir o desconforto respiratório e a hipoxemia foi a Ventilação Mecânica Não Invasiva (VNI) intermitente com máscara facial total (Performax Respironics $^{\circledR}$ ), intercalando com máscara reservatório de $\mathrm{O}_{2}$. A paciente não se adaptou a tais dispositivos, relatando incomodo principalmente em relação à VNI. Com o objetivo de diminuir esse incomodo foram testados outros tipos interfaces, porém não houve adesão.

Durante o uso da máscara reservatório de $\mathrm{O}_{2}$ com fluxo de 15 l/min apresentou taquipneia com Frequência Respiratória (f) igual a 40rpm, hipossaturação com Saturação Periférica de Oxigênio $\left(\mathrm{SpO}_{2}\right)$ de $58 \%$, taquicardia com Frequência Cardíaca (FC) de 135 bpm, pico hipertensivo com Pressão Arterial (PA) 175x85 mmHg. A gasometria arterial revelou hipoxemia grave e Saturação arterial de Oxigênio $\left(\mathrm{SaO}_{2}\right)$ abaixo dos níveis de normalidade (Tabela 1). A equipe então resolveu instalar o Alto Fluxo Nasal (AFN) com o sistema 


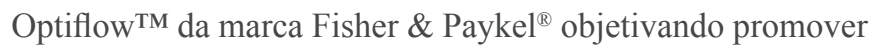
maior conforto à paciente.

Foi utilizado fluxo de $30 \mathrm{l} / \mathrm{min}$ de $\mathrm{O}_{2}$ sem mistura de ar comprimido com $\mathrm{FiO}_{2}$ de $100 \%$ e temperatura de $37^{\circ} \mathrm{C}$. Após 30 minutos de utilização, avaliou-se os sinais vitais, obtendo-se os seguintes resultados: $\mathrm{f}=30 \mathrm{rpm}, \mathrm{FC}=127 \mathrm{bpm}, \mathrm{PA}=156 \times 69$ $\mathrm{mmHg}, \mathrm{SpO}_{2}=80 \%$, aumento da $\mathrm{SaO}_{2}$, redução da hipoxemia e do uso de musculatura acessória e a paciente referiu maior conforto (Tabela 1). Também foi possível observar melhores resultados gasométricos com 24 horas de uso da terapia (Tabela 1). Os níveis de $\mathrm{SpO}_{2}$ estão melhor representados no gráfico 1 onde é possível visualizar a curva ascendente da saturação após o uso da terapia com AFN (Gráfico 1).

Tabela 1. Dados gasométricos com máscara reservatório e AFN.

\begin{tabular}{|c|c|c|}
\hline $\begin{array}{l}\text { Máscara } \\
\text { reservatório }\end{array}$ & $\begin{array}{l}30^{\prime} \text { com Alto Fluxo } \\
\text { Nasal } 301 / \text { min }\end{array}$ & $\begin{array}{l}\text { Após } 24 \text { horas com } \\
\text { Alto Fluxo Nasal } \\
301 / \mathrm{min}\end{array}$ \\
\hline *pH: 7,44 & pH: 7,44 & pH: 7,43 \\
\hline$* \mathrm{PaCO}_{2}: 47 \mathrm{mmHg}$ & $\mathrm{PaCO}_{2}: 46 \mathrm{mmHg}$ & $\mathrm{PaCO}_{2}: 43 \mathrm{mmHg}$ \\
\hline$* \mathrm{PaO}_{2}: 36 \mathrm{mmHg}$ & $\mathrm{PaO}_{2}: 46 \mathrm{mmHg}$ & $\mathrm{PaO}_{2}: 56 \mathrm{mmHg}$ \\
\hline$* \mathrm{HCO}_{3}: 31.9 \mathrm{mmol} / \mathrm{L}$ & $\mathrm{HCO}_{3}: 31.2 \mathrm{mmol} / \mathrm{L}$ & $\mathrm{HCO}_{3}: 35 \mathrm{mmol} / \mathrm{L}$ \\
\hline$* \mathrm{SaO}_{2}: 72 \%$ & $\mathrm{SaO}_{2}: 83 \%$ & $\mathrm{SaO}_{2}: 90 \%$ \\
\hline *BE: $6.7 \mathrm{mmol} / \mathrm{L}$ & BE: $6.1 \mathrm{mmol} / \mathrm{L}$ & BE: $9.2 \mathrm{mmol} / \mathrm{L}$ \\
\hline
\end{tabular}

*pH- Potencial de Hidrogênio; PaCO2- Pressão arterial de gás carbônico; $\mathrm{PaO} 2$ - Pressão arterial de oxigênio; HCO3- Bicarbonato; $\mathrm{SaO} 2-$ Saturação arterial de oxigênio; BE- Base Excess.

Gráfico 1. Níveis de Saturação Periférica de Oxigênio (SpO2) com máscara reservatório e AFN.

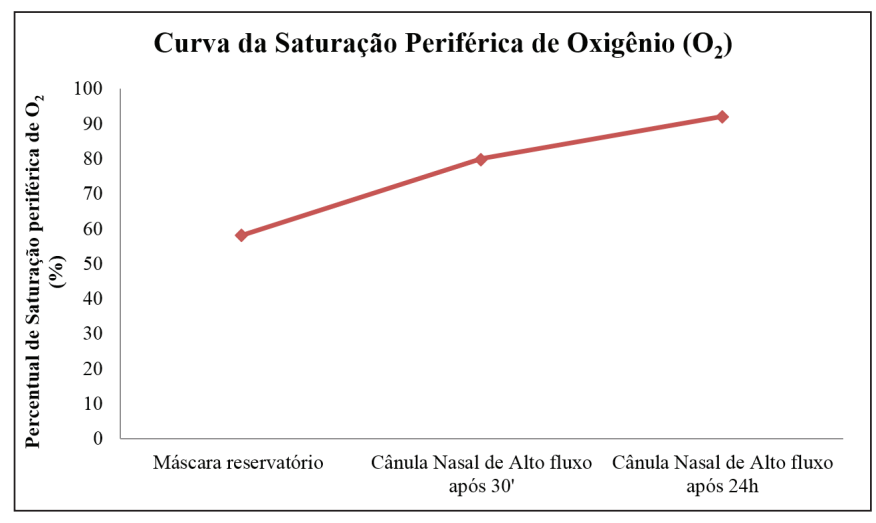

Após sete dias de uso a paciente foi a óbito. O AFN trouxe conforto para a mesma em seus últimos momentos permitindo que houvesse interação com a família e realização de atividades simples como dormir e se alimentar, proporcionando assim uma morte humanizada e tranquila.

\section{DISCUSSÃO}

Os resultados encontrados neste relato de caso sugerem o benefício do uso do AFN em uma paciente com FPI em cuidados paliativos, onde foi possível observar uma redução do desconforto respiratório e da hipoxemia, além de permitir interação com a família e a realização de atividades diárias, proporcionando conforto e tranquilidade no fim da vida.

Corroborando com o presente estudo, Calvano e Cols em 2008 publicaram um relato de caso de uma mulher de 92 anos em cuidados paliativos com demência e pneumonia multilobar, a mesma era incapaz de tolerar máscaras faciais e nasais utilizadas em VNI, apresentando hipoxemia em uso de máscara de Venturi 50\%. Utilizou-se o AFN com 30 1/min e como resultados verificou-se redução da f de 35 para $23 \mathrm{rpm}$, FC de 106 para $80 \mathrm{bpm}, \mathrm{SpO}_{2}$ aumentou de $80 \%$ para $98 \%$ e $\mathrm{PaO}_{2}$ de 39 para $64 \mathrm{mmHg}$. A paciente tornou-se mais relaxada e cooperativa, iniciou dieta regular, melhorou a dispneia, oxigenação e tolerância a oxigenoterapia, promovendo maior conforto no fim da vida. ${ }^{8}$

A dificuldade de tolerar máscaras faciais de VNI foi observada na paciente deste relato, além disso, não foi possível manter uma oxigenação adequada com outros dispositivos de oxigenoterapia.

Um estudo publicado em 2015 comparou terapia padrão com oxigênio, AFN e VNI em pacientes com IRpA hipoxêmica, mostrou que a dispneia foi menor com o uso do AFN, resultando assim na redução da mortalidade na UTI em 90 dias nos pacientes que utilizaram AFN quando comparado aos grupos de terapia padrão e VNI. ${ }^{9}$

O AFN também se mostrou eficaz em um estudo piloto realizado em 2013, aumentando o volume corrente em pacientes com Doença Pulmonar Obstrutiva Crônica (DPOC), além de reduzir a frequência respiratória e volume minuto nesses pacientes, em pacientes com FPI e em voluntários saudáveis. Nesse estudo a Pressão Arterial de Gás Carbônico $\left(\mathrm{PaCO}_{2}\right)$ diminuiu nos pacientes com FPI e DPOC, resultando em efeitos significativos sobre parâmetros respiratórios em pacientes com doença pulmonar obstrutiva e restritiva contribuindo para o aumento da eficácia de ventilação e redução do trabalho de respiratório. ${ }^{10}$

A interação da paciente com a família também foi um fator importante para o conforto da mesma, permitindo a comunicação e expressão de seus sentimentos até os seus últimos momentos. Achados semelhantes foram descritos por Mahler e cols, onde observaram que a terapia com AFN permitiu uma melhor interação com as famílias, pois melhorou significativamente a oxigenação e reduziu a tosse de pacientes em fase terminal com hipoxemia grave decorrente de doenças pulmonares intersticiais, quando comparada às máscaras de oxigênio sem reinalação. ${ }^{11}$

Os resultados do presente estudo mostraram melhora considerável na oxigenação após o AFN. Sztrymf e cols, após o uso do AFN e oxigenoterapia convencional em 20 pacientes com IRpA persistente ao uso de oxigênio observaram que a oxigenoterapia por AFN permitiu uma redução da $\mathrm{f}$ (28 para 24,5 rpm), aumento significativo da $\mathrm{PaO}_{2}(8,73$ a 15,27) e da $\mathrm{SpO}_{2}(93,5 \%$ para 98,5\%). Deste modo a terapia de AFN de 
oxigênio em pacientes com IRpA persistente, está associada à melhora significativa e sustentada dos parâmetros clínicos e biológicos. ${ }^{12}$

$\mathrm{Na}$ emergência de um Hospital Universitário Francês, compararam a cânula de AFN e a máscara reservatório em pacientes com IRpA. Os resultados mostraram que a terapia com AFN resultou na redução da $\mathrm{f}(\mathrm{p}<0,05)$ e dos sinais de desconforto respiratório $(\mathrm{p}<0,05)$, aumento da $\mathrm{SpO}_{2}(\mathrm{p}<0,01)$, melhora rápida e sustentada da dispneia, além de ser bem tolerado e mais confortável do que terapia convencional de oxigênio. ${ }^{13}$

O conforto desta terapia ocorre principalmente devido a umidificação oferecida, sendo este avaliado em 30 pacientes

\section{REFERÊNCIAS}

1. American Thoracic Society; European Respiratory Society. This joint statement of the American Thoracic Society (ATS), and the European Respiratory Society (ERS) was adopted by the ATS board of directors, June 2001 and by the ERS Executive Committee, June 2001. Am J Respir Crit Care Med. 2002;165(2):277-304. Erratum in: Am J Respir Crit Care Med. 2002;166(3):426.

2. Spruit MA, Janssen DJ, Franssen FM, Wouters EF. Rehabilitation and palliative care in lung fibrosis. Respirology. 2009;14(6):781-7.

3. Lanken PN, Terry PB, Delisser HM, Fahy BF, Hansen-Flaschen J, Heffner JE, et al. An official American Thoracic Society clinical policy statement: palliative care for patients with respiratory diseases and critical illnesses. Am J Respir Crit Care Med. 2008;177(8):912-27.

4. Carvalho RT, Parsons HA (organizadores). Manual de cuidados paliativos ANCP. Rio de Janeiro: Diagraphic; 2009. Capítulo 2, Dispnéia, tosse e hipersecreção de vias aéreas; p. 104-13.

5. Bartholo TP, Gomes MM, Noronha AJ Filho. DPOC: o impacto da oxigenoterapia domiciliar no tratamento. Pulmão RJ. 2009;1(1);7984.

6. Cachia E, Ahmedzai SH. Breathlessness in cancer patients. Eur J Cancer. 2008;44(8):1116-23.

7. Gotera C, Díaz Lobato S, Pinto T, Winck JC. Clinical evidence on high flow oxygen therapy and active humidification in adults. Rev Port Pneumol. 2013;19(5):217-27.

8. Calvano TP, Sill JM, Kemp KR, Chung KK. Use of a high-flow com IRpA hipoxêmica através da comparação da terapia de oxigênio padrão com o AFN aquecido e umidificado. $\mathrm{O}$ ressecamento nasal foi significativamente menor no grupo AFN em 4hs $(p=0,007)$ e $24 \mathrm{hs}(\mathrm{p}=0,004)$, além disso, obteve maior preferência dentre os participantes $(p=0,01)$, sobretudo aqueles que necessitaram de fluxos mais altos de oxigênio no momento da internação $(p=0,05) \cdot{ }^{14}$

\section{CONCLUSÃO}

O AFN foi um fator importante para a melhora da oxigenação e conforto da paciente do caso relatado, garantindo além da melhora clínica, uma morte tranquila com a redução dos sintomas respiratórios que são angustiantes para o paciente, para família e para a equipe.

oxygen delivery system in a critically ill patient with dementia. Respir Care. 2008;53(12):1739-43.

9. Frat JP, Thille AW, Mercat A, Girault C, Ragot S, Perbet S, et al. High-flow oxygen through nasal cannula in acute hypoxemic respiratory failure. N Engl J Med. 2015;372:2185-96.

10. Bräunlich J, Beyer D, Mai D, Hammerschmidt S, Seyfarth HJ, Wirtz H. Effects of nasal high flow on ventilation in volunteers, COPD and idiopathic pulmonary fibrosis patients. Respiration. 2013;85(4):319-25

11. Mahler DA, Selecky PA, Harrod CG, Benditt JO, CarrieriKohlman V, Curtis JR, et al. American College of Chest Physicians consensus statement on the management of dyspnea in patients with advanced lung or heart disease. Chest. 2010;137(3):674-91.

12. Sztrymf B, Messika J, Mayot T, Lenglet H, Dreyfuss D, Ricard JD. Impact of high-flow nasal cannula oxygen therapy on intensive care unit patients with acute respiratory failure: a prospective observational study. J Crit Care. 2012;27(3):324.e9-13.

13. Lenglet H, Sztrymf B, Leroy C, Brun P, Dreyfuss D, Ricard JD. Humidified high flow nasal oxygen during respiratory failure in the emergency department: feasibility and efficacy. Respir Care. 2012;57(11):1873-8.

14. Cuquemelle E, Pham T, Papon JF, Louis B, Danin PE, Brochard L. Heated and humidified high-flow oxygen therapy reduces discomfort during hypoxemic respiratory failure. Respir Care. 2012;57(10):1571-7.

\section{Como citar:}

Silva FS, Barreto PH, Vasconcelos RS, Sales RP, Nogueira AN, Holanda MA. Oxigenoterapia de alto fluxo nasal em paciente com fibrose pulmonar idiopática sob cuidados paliativos: relato de um caso. Rev Med UFC. 2017 jan-abr;57(1):69-72. 